\title{
The Composition of Late-Accreted Planetesimals
}

\author{
P. J. CARTER* AND S. T. STEWART
}

Department of Earth and Planetary Sciences, University of California, Davis, One Shields Avenue, Davis, CA 95616

(*correspondence: pjcarter@ucdavis.edu)

The presence of highly siderophile elements in Earth's mantle indicates that a small percentage of the Earth's mass was delivered after the last giant impact and closure of Earth's core in a stage of 'late accretion.' There is substantial debate about the nature of this late accreted material, and the sizes of the bodies that delivered it. Earth appears isotopically most similar to the enstatite chondrite family, and it has been suggested that late accretion must also have been dominated by enstatite-like material, rather than carbonaceous chondrites. The origin of late accreted bodies is unknown, but the lack of a substantial outer solar system isotopic signature suggests that most late-accreted mass originated in the inner disk.

Planet formation models typically show continued planetesimal impacts at late times, but these planetesimals are usually unable to evolve during the simulations. Here, we use high-resolution, fully interacting, collisional $N$-body simulations that allow us to follow the evolution and production (via fragmentation) of small bodies. We examine the compositions (defined by initial locations) of the 'leftover planetesimals' in the late stages of these accretion simulations.

Dynamically excited planet formation produces Earth-like planets with a mixture of material from across the inner solar system. This mixing of initially separated reservoirs is expected to lead to mixing of material with distinct isotopic signatures. There are several embryos remaining at the end of these simulations, many of which have similar compositions. These final bodies include smaller embryos that are very similar in composition to the Earth-sized planets, suggesting that Theia may have had a similar stable isotope composition to the proto-Earth. Planetesimal-sized bodies with similar compositions to the Earth-like planets are also present at the end of the main period of accretion. Many of these chemically similar leftover planetesimals are silicate or iron-rich fragments of proto-planets ejected millions of years earlier.

Embryos and planetesimals become similar early (in the first few Myr) in the Grand Tack scenario. There are thus many intermediate bodies with similar compositions early in the giant impact phase. The final planets can have similar compositions to several intermediate embryos, and planetesimal-sized fragments can be ejected from any of these embryos. If these fragments are later accreted by the planets, they would represent late accreted mass with isotopic compositions that naturally match the composition of the planet. 